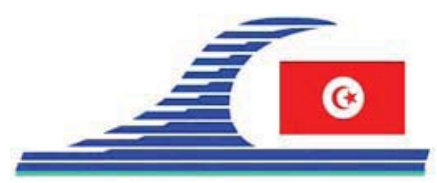

Conférence Méditerranéenne Côtière et Maritime EDITION 1, HAMMAMET, TUNISIE (2009)

Coastal and Maritime Mediterranean Conference

Disponible en ligne - http://www.paralia.fr-Available online

\title{
Modélisation de la turbidité en Baie de Bourgneuf (France)
}

\author{
Morgan DUSSAUZE ${ }^{1}$, Martin SANCHEZ ${ }^{1}$, Laurent BARILLÉ $^{2}$, \\ Benoist HITIER $^{3}$, Hélène OGER-JEANNERET ${ }^{3}$, Marc ROBIN $^{4}$
}

1. Université de Nantes, UMR 6112 CNRS-LPG, 2 rue de la Houssinière, BP 92208, 44322 Nantes, France.morgan.dussauze@univ-nantes.fr

2. Université de Nantes, Mer Molécules Santé UPRES EA 2160, Nantes, France.

3. Ifremer, Laboratoire Environnement-Ressources Morbihan-Pays de Loire, rue Ile d'Yeu, 44300 Nantes, France.

4. Université de Nantes, UMR 6554 CNRS - LETG, Château du Tertre, BP 81227, 44312 Nantes Cedex, France.

\section{Résumé :}

Un modèle numérique est mis en place afin de modéliser la turbidité en Baie de Bourgneuf (France) à partir d'une couverture réaliste de sédiments fins. Trois agents hydrodynamiques sont pris en compte : les courants de marée; la houle se propageant des grandes profondeurs vers la côte et le clapot généré localement par le vent. Cette modélisation permet de représenter le gradient Nord/Sud de turbidité, et les rapides changements de turbidité qui sont mesurés au niveau de la zone intertidale au cours d'un cycle de marée et cela en accord avec les mesures in situ effectuées sur l'estran et les mesures satellites.

\section{Mots-clés :}

Sédiment fin - Marée - Houle - Erosion - Transport en suspension - Turbidité

\section{Introduction}

Située au sud de l'estuaire de la Loire, la Baie de Bourgneuf est un bassin ostréicole d'une grande importance. Les performances de croissance des huîtres y sont cependant sensiblement inférieures aux autres sites ostréicoles français notamment en raison de l'état général des concessions (HAURE et al., 2003), de la compétition trophique due aux huîtres sauvages et aux crépidules, et enfin de la turbidité élevée, qui altère les fonctions écophysiologiques de l'huître et entraîne ainsi une diminution de la qualité de la nourriture ingérée (BARILLÉ et al., 1997). Dans ce contexte, l'axe 3 du projet GERRICO (co-direction Ifremer et Université de Nantes) vise à développer une méthodologie permettant de prendre en compte, par l'intermédiaire d'une chaîne de modèles couplés, l'ensemble d'un espace depuis les bassins versants jusqu'à la mer côtière. Cette méthodologie va permettre, entre autres, d'aboutir à une modélisation de la croissance des huîtres. Une bonne représentation de cette croissance est dépendante d'une bonne modélisation de la turbidité au niveau des zones de cultures. Ainsi, un 
modèle numérique de transport des sédiments fins en baie de Bourgneuf est mis en place à l'aide du logiciel MIKE 21 afin de reproduire de manière réaliste les agents hydrodynamiques (courants de marée, houle du large et surtout clapot généré localement par le vent) responsables de la remise en suspension du sédiment fin présent en fond de baie. Ce travail est la continuité de l'étude visant à expliquer les accumulations de sédiments fins dans certaines zones côtières non estuariennes (SANCHEZ, 2008).

\section{Site d'étude}

La Baie de Bourgneuf est une zone séparée de l'océan par l'île de Noirmoutier et par conséquent bien abritée par rapport à la houle. Le panache de la Loire, dont l'estuaire se trouve à proximité, se dirige de manière générale vers le nord et pénètre rarement dans la baie. Cependant, par vent de nord, la Loire l'influence de manière importante. Les sédiments fins en général y sont présents dans des proportions allant de 1 à $50 \%$ (GOULEAU, 1968). La baie est caractérisée par la présence de vase qu'on trouve principalement dans sa partie orientale. Dans cette même zone, une accumulation de "crème de vase", facilement mobilisable, couvre une surface d'environ 2000 hectares. En condition de tempête, ce sédiment fin est remis en suspension par la houle et le clapot entraînant ainsi une très forte turbidité, notamment au niveau de l'estran, dépassant largement les $200 \mathrm{mg} \mathrm{L}^{-1}$.

\section{Méthodes}

Le logiciel utilisé est Flow Model MIKE 21 (DHI, 2007a) qui permet de calculer, en chaque maille du domaine (figure 1) l'élévation de la surface libre et les courants de marée à partir de conditions aux frontières calculées selon une méthode des constantes harmoniques. La propagation de la houle est calculée séparément par le module Spectral Waves MIKE 21 (DHI, 2007b), après validation de la marée à l'aide des marégrammes de Saint-Nazaire, de mesures effectuées sur l'estran en 2005 et 2006 ainsi qu'un atlas de courant (SHOM, 1990). La propagation de houle est également validée à l'aide de mesures à l'Ile d'Yeu $\left(02^{\circ} 25.65^{\prime} \mathrm{W}, 47^{\circ} 41.45^{\prime} \mathrm{N}\right)$ issues du réseau CANDHIS (site web) du CETMEF. Le modèle simule l'érosion et le dépôt, respectivement selon les formulations de PARTHENIADES (1965) et de KRONE (1986), sur quelques jours à partir d'une couverture de vase inspirée d'une carte sédimentologique de la baie (GOULEAU, 1968). Le débit de la Loire n'est pas pris en compte ; l'intérêt est porté, dans un premier temps, uniquement à la remise en suspension du sédiment présent sur l'estran. La turbidité modélisée est confrontée à des images SPOT à partir desquelles ont été quantifiées les matières en suspensions. L'empiric line correction (SMITH \& MILTON, 1999) est utilisée afin de transformer les comptes numériques en réflectance puis une relation logarithmique (FROIDEFOND et al., 2003) est appliquée pour passer de la réflectance à la valeur de turbidité. 


\section{Résultats}

La figure 2 représente la turbidité modélisée comparée à celle issue de l'imagerie satellitaire (LEROUXEL et al., 2007) le 13/12/2005 environ 2 heures avant l'étale de haute mer (coefficient de marée 73). Ce jour correspond à des conditions de houle et de vent relativement faibles (hauteur significative de houle inférieure à $1 \mathrm{~m}$ à l'île d'Yeu, vitesse du vent d'environ $10 \mathrm{~m} \mathrm{~s}^{-1}$ ). Le modèle reproduit de manière correcte aussi bien le panache turbide que son intensité (concentrations supérieures à $200 \mathrm{mg} \mathrm{L}^{-1}$ dans la partie orientale de la baie; au maximum $100 \mathrm{mg} \mathrm{L}^{-1}$ près de Noirmoutier). Les conditions hydrodynamiques modélisées (hauteur significative de houle d'environ $10 \mathrm{~cm}$ au niveau de l'estran) permettent la remise en suspension du sédiment qui, couplée avec de faibles hauteurs d'eau, entraîne de très fortes turbidités.

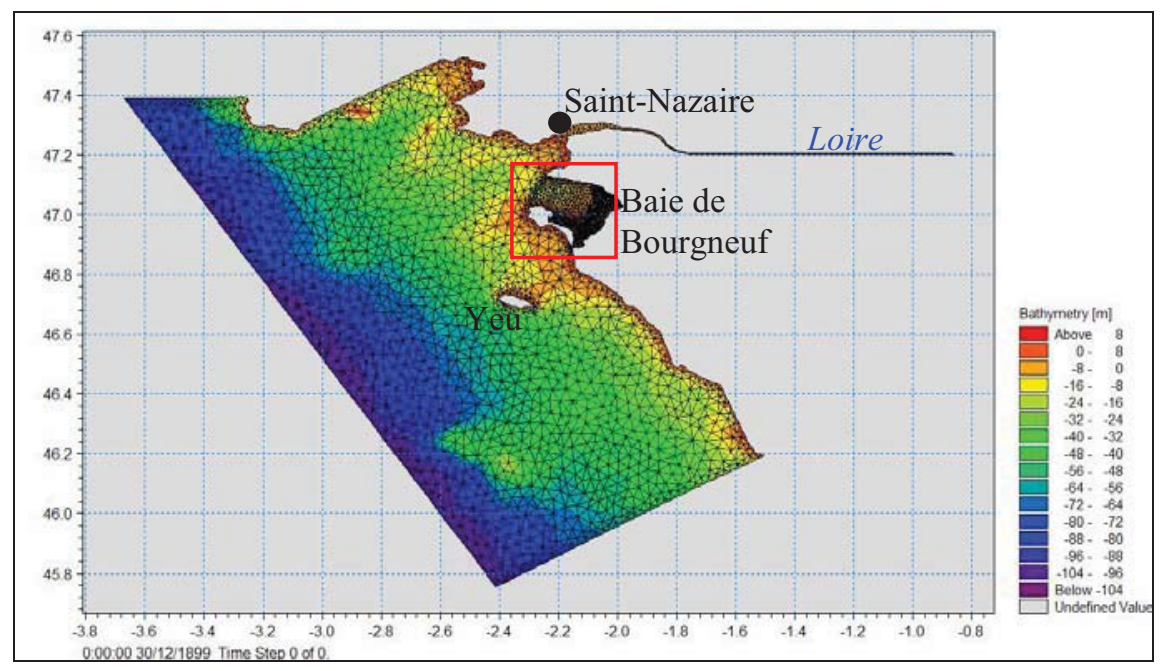

Figure 1. Maillage du domaine d'étude sur fond de bathymétrie (mailles de $3 \mathrm{~km} \mathrm{au}$ large et 300 m au niveau de la Baie de Bourgneuf).

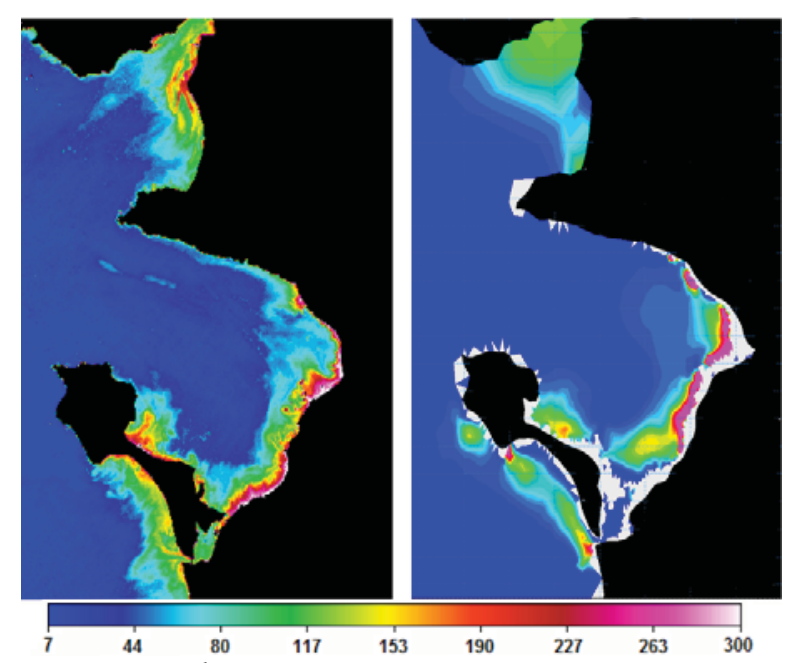

Figure 2. Turbidité ( $m g L^{-1}$ ) en Baie de Bourgneuf (à gauche image SPOT du $13 / 12 / 2005$ à $11 \mathrm{~h}$; à droite résultats du modèle le même jour à la même heure). 


\section{Conclusion}

Ce travail a permis de représenter le panache turbide observé en baie de Bourgneuf à l'aide du modèle MIKE 21. Une meilleure représentation du clapot nécessiterait une prise en compte du vent et une bathymétrie plus fine (PARISOT et al., 2008) ainsi que des mesures sur l'estran permettant de valider les résultats. Cependant, les résultats obtenus sont suffisants pour pouvoir les associer en tant que forçage ou couplage à un modèle de croissance d'huîtres dans les différents secteurs de la baie. Ainsi, l'acquisition d'informations spatialisées constituera un outil pertinent d'aide à la gestion des espaces conchylicoles, permettant en particulier une analyse plus précise des éventuelles restructurations des bancs ostréicoles.

\section{Remerciements}

Le projet GERRICO est piloté par l'Ifremer et l'Université de Nantes, avec le soutien de la Région des Pays de la Loire. Les auteurs remercient ces trois institutions.

\section{Références bibliographiques}

BARILLÉ L., HERAL M., BARILLÉ-BOYER A.L. (1997). Modélisation de l'écophysiologie de l'huître Crassostrea gigas dans un environnement estuarien. Aquat. Living Resour. Vol. 10, nº 1, pp 31-48.

CANDHIS (site web). http://candhis.cetmef.developpement-durable.gouv.fr/

DHI (2007a). MIKE 21 \& MIKE 3 flow model FM. Hørsholm, Denmark, 14 p.

DHI (2007b). MIKE 21 wave modelling. Hørsholm, Denmark, 16 p.

FROIDEFOND J.-M., DOXARAN D., MILLER P. (2003). Acquisition et traitement d'images satellites. Programme interrégional Loire grandeur nature, GIP Loire estuaire, Nantes, 174 p.

GOULEAU D. (1968). Etude hydrologique et sédimentologique de la baie de Bourgneuf. Thèse de $3^{\text {ème }}$ cycle, Université de Nantes, 365 p.

HAURE J., MARTIN J.L.Y., DUPUY B., NOURRY M., PALVADEAU H., PAPIN M., PENISSON C., LE MOINE O., GEAIRON P., RAZET D. (2003). Estimation des stocks d'huîtres en élevage dans la Baie de Bourgneuf en 2002. Restitution des travaux 2002, contrat Région pays de la Loire, contrat Conseil Général de Vendée et SRC Pays de Loire, DRV/RA/LCPL, 19 p.

KRONE R.B. (1986). The significance of aggregate properties to transport processes. In A.J. Mehta (Ed.), Estuarine cohesive sediment dynamics, Coastal and estuarine studies $n^{\circ} 14$ (pp 66-84). Springer, Berlin.

LEROUXEL A., BARILlÉ L., ROSA P., FROIDEFOND J.M. (2007). Analyse de la distribution spatiale de la turbidité en baie de Bourgneuf et de son impact sur les cultures ostréicoles. Étude accordée par le SMIDAP (Syndicat Mixte pour le Développement de l'Aquaculture et de la Pêche en Pays de la Loire). 52 p.

PARISOT J.P., DIET-DAVANCES J., SOTTOLICHIO A., CROSLAND E., DRILLON C., VERNEY R. (2008). Modélisation des agitations dans le bassin d'Arcachon. Xèmes Journées Nationales Génie Côtier - Génie Civil, Sophia Antipolis, pp 435-444. [URL http://www.paralia.fr/jngcgc/10_42_parisot.pdf] PARTHENIADES E. (1965). Erosion and deposition of cohesive soils. Journal of the Hydraulic Division, $\mathrm{n}^{\circ}$ 91, pp 105-139.

SANCHEZ M. (2008). Dynamique des sédiments fins dans une zone côtière à forte turbidité à proximité de l'embouchure de la Loire. Xèmes Journées Nationales Génie Côtier - Génie Civil, Sophia Antipolis, pp 169-178. [URL http://www.paralia.fr/jngcgc/10_16_sanchez.pdf ]

SHOM (1990). Courants de marée de la côte sùd de Bretagne de Penmarc'h à Noirmoutier. Service hydrographique et océanographique de la marine, Paris, $48 \mathrm{p}$.

SMITH G.M., MILTON E.J. (1999). The use of the empirical line method to calibrate remotely sensed to reflectance. Int. J. Remote Sens. 20 (13), pp 2653-2662. 